\title{
Subprincipal symbol for Toeplitz operators
}

\author{
Laurent Charles *
}

October 22, 2018

\begin{abstract}
We establish some subprincipal estimates for Berezin-Toeplitz operators on symplectic compact manifolds. From this, we construct a family of subprincipal symbol maps and we prove that these maps are the only ones satisfying some expected conditions.

Keywords: Berezin-Toeplitz operators, subprincipal symbol, Quantization, Kähler manifolds, symplectic manifolds. MSC: 53D50, 81S10
\end{abstract}

Toeplitz operators on symplectic manifolds are similar to semiclassical pseudo-differential operators on cotangent bundles. In particular, we can extend to Toeplitz operators the usual techniques to describe the spectrum of pseudodifferential operators, as for instance the trace formula BPU98 or the BohrSommerfeld conditions Cha03b, Cha06, Le 13. Two important ingredients in these semi-classical results are the principal and subprincipal symbols of an operator. One issue is that there is no obvious definition for the subprincipal symbol of a Toeplitz operators, cf. as instance BPU98 or BdM02.

In this paper, we introduce some axioms that a subprincipal symbol should satisfy to our point of view. Then we construct all the subprincipal symbol satisfying these axioms. We work in the general setting introduced in Cha15 for the quantization of symplectic manifolds. General reference for the Toeplitz operators are BdMG81, MM07, BMS94, Gui95. Our construction is inspired from our previous work on Kähler manifolds, in particular Cha06 and Cha07, and uses in an essential way the metaplectic correction.

\section{Statement of the results}

To start with, consider a compact Kähler manifold $M$ equipped with a holomorphic Hermitian line bundle $L$ and with a holomorphic Hermitian vector bundle $A$. Assume that $L$ is positive so that the Chern curvature of $L$ is $\frac{1}{i} \omega$ where $\omega \in \Omega^{2}(M, \mathbb{R})$ is symplectic. For any $k \in \mathbb{N}$, let $\mathcal{H}_{k}$ be the space of holomorphic sections of $L^{k} \otimes A$.

\footnotetext{
*Sorbonne Universités, UPMC Univ Paris 06, UMR 7586, Institut de Mathématiques de Jussieu-Paris Rive Gauche, F-75005, Paris, France
} 
More generally, let $(M, \omega)$ be any symplectic compact manifold such that $\frac{1}{2 \pi}[\omega]$ is integral. Introduce a Hermitian line bundle $L \rightarrow M$ with a Hermitian connection $\nabla$ of curvature $\frac{1}{i} \omega$, a Hermitian vector bundle $A$ and an almost complex structure $j$ compatible with $\omega$. Then consider a family of finite dimensional subspace

$$
\mathcal{H}_{k} \subset \mathcal{C}^{\infty}\left(M, L^{k} \otimes A\right), \quad k \in \mathbb{N}^{*}
$$

consisting of almost-holomorphic sections in the sense of Cha15. In the sequel, we will refer to the case where $M$ is Kähler, $L$ and $A$ holomorphic, $\nabla$ is the Chern connection and $\mathcal{H}_{k}$ consists of holomorphic sections, as the Kähler case. Observe that $\mathcal{H}_{k}$ has a natural scalar product obtained by integrating the pointwise scalar product against the Liouville measure $\omega^{n} / n !$.

To these data is associated a star-algebra $\mathcal{T}$ consisting of the so-called Berezin-Toeplitz operators or more briefly Toeplitz operators. The definition will be recalled later, let us just say for now that a Toeplitz operator $T$ is an endomorphism family $\left(T_{k}: \mathcal{H}_{k} \rightarrow \mathcal{H}_{k}, k \in \mathbb{N}\right)$. The product of $\mathcal{T}$ is the usual composition of endomorphisms and the involution is the Hermitian adjoint. A very important fact is the existence of a natural star-algebra morphism $\sigma_{\mathrm{p}}: \mathcal{T} \rightarrow \mathcal{C}^{\infty}(M$, End $A)$ which is onto and with kernel

$$
k^{-1} \mathcal{T}:=\left\{\left(k^{-1} T_{k}\right) /\left(T_{k}\right) \in \mathcal{T}\right\}=\left\{\left(T_{k}\right) \in \mathcal{T} /\left\|T_{k}\right\|=\mathcal{O}\left(k^{-1}\right)\right\} .
$$

For any $T \in \mathcal{T}, \sigma_{\mathrm{p}}(T)$ is called the principal symbol of $T$. Furthermore, if $T$ and $S$ are two Toeplitz operators with scalar valued principal symbol $f$ and $g$ respectively, then $i k[T, S]$ is a Toeplitz operator with principal symbol $\{f, g\}$, where $\{\cdot, \cdot\}$ is the Poisson bracket corresponding to $\omega$.

Let $\mathcal{T}_{\text {sc }}$ be the subalgebra of $\mathcal{T}$ consisting of operators with a scalar valued principal symbol. We say that a linear map $\sigma_{\mathrm{s}}: \mathcal{T}_{\mathrm{sc}} \rightarrow \mathcal{C}^{\infty}(M$, End $A)$ is a subprincipal symbol map if it satisfies the following conditions:

i) for any $T \in \mathcal{T}, \sigma_{\mathrm{s}}\left(k^{-1} T\right)=\sigma_{\mathrm{p}}(T)$,

ii) for any $T \in \mathcal{T}_{\mathrm{sc}}, \sigma_{\mathrm{s}}\left(T^{*}\right)=\sigma_{\mathrm{s}}(T)^{*}$,

iii) for any $T, S \in \mathcal{T}_{\mathrm{sc}}, \sigma_{\mathrm{s}}(T S)=\sigma_{\mathrm{p}}(T) \sigma_{\mathrm{s}}(S)+\sigma_{\mathrm{s}}(T) \sigma_{\mathrm{p}}(S)+\frac{1}{2 i}\left\{\sigma_{\mathrm{p}}(T), \sigma_{\mathrm{p}}(S)\right\}$,

With such a map $\sigma_{\mathrm{s}}$, we can control the Toeplitz operators with a scalar principal symbol up to $k^{-2} \mathcal{T}$. More precisely, by i) and ii), the map

$$
\sigma:=\sigma_{\mathrm{p}}+\hbar \sigma_{\mathrm{s}}: \mathcal{T}_{\mathrm{sc}} \rightarrow \mathcal{C}^{\infty}(M) \oplus \hbar \mathcal{C}^{\infty}(M, \text { End } A)
$$

is real, onto with kernel $k^{-2} \mathcal{T}_{\text {sc }}$.

As a first attempt to construct a subprincipal symbol map, we can consider the contravariant symbols. Let $\Pi_{k}$ be the orthogonal projector of $\mathcal{C}^{\infty}\left(M, L^{k} \otimes A\right)$ onto $\mathcal{H}_{k}$. Recall that for any $f \in \mathcal{C}^{\infty}(M$, End $A),\left(\Pi_{k} f, k \in \mathbb{N}\right)$ is a Toeplitz operator with principal symbol $f$. So by the properties of the principal symbol recalled above, for any Toeplitz operator $T \in \mathcal{T}$, we have $T=\Pi f \bmod k^{-1} \mathcal{T}$ with $f=\sigma_{\mathrm{p}}(T)$. We can now set $\sigma_{\mathrm{s}}^{\mathrm{c}}(T):=\sigma_{\mathrm{p}}(k(T-\Pi f))$, so that we have

$$
T=\Pi f+k^{-1} \Pi g \quad \bmod k^{-2} \mathcal{T} \quad \text { with } f=\sigma_{\mathrm{p}}(T), g=\sigma_{\mathrm{s}}^{\mathrm{c}}(T)
$$


This defines a map $\sigma_{\mathrm{s}}^{\mathrm{c}}$ which satisfies i) ii) but not iii). For instance, in the Kähler case with $A$ the trivial line bundle, we proved in Cha03a that $\sigma_{\mathrm{s}}^{\mathrm{c}}(S T)=$ $\sigma_{\mathrm{p}}(T) \sigma_{\mathrm{s}}^{\mathrm{c}}(S)+\sigma_{\mathrm{s}}^{\mathrm{c}}(T) \sigma_{\mathrm{p}}(S)+\hbar B(\sigma(S), \sigma(T))$ where in complex coordinates $\left(z^{i}\right)$,

$$
B(f, g)=-\sum G^{j k} \partial_{z_{j}} f . \partial_{\bar{z}_{k}} g \quad \text { if } \quad \omega=i \sum G_{j, k} d z_{j} \wedge d \bar{z}_{k} .
$$

As expected, the antisymmetric part of $B$ is $\frac{1}{2 i}$ times the Poisson bracket. But the symmetric part does not vanish. Nevertheless, setting

$$
\sigma_{\mathrm{s}}(T)=\sigma_{\mathrm{s}}^{\mathrm{c}}(T)+\frac{\hbar}{2} \Delta \sigma_{\mathrm{p}}(T)
$$

where $\Delta=\sum G^{j k} \partial_{z_{j}} \partial_{\bar{z}_{k}}$, we obtain a subprincipal symbol map, which was introduced in Cha03b to state the Bohr-Sommerfeld conditions. Equation (2) may be viewed as a generalization to Kähler manifolds of the formula giving the subprincipal term of the Weyl symbol in terms of the anti-Wick symbol. Still in the Kähler case, this approach can be generalized to any holomorphic vector bundle $A$ by using the expression for $B$ obtained in MM12. In the general symplectic case, we only know that $B$ is a bidifferential operator. Using some standard argument in deformation quantization, this is enough to deduce the existence of a subprincipal symbol map, cf. Proposition 3.2. But we do not have an explicit formula defining this symbol.

Actually, there is a direct way to define an explicit subprincipal symbol. The construction is easier in the case where the canonical bundle $K=\wedge^{n, 0} T^{*} M$ has a square root $(\delta, \varphi)$, what we assume from now on. Here $\delta$ is a Hermitian line bundle over $M$ and $\varphi$ is an isomorphism from $\delta^{2}$ to $K$. It is known that such a square root exists if and only if $M$ has a spin structure if and only if the second Stiefel-Withney class of $M$ vanishes.

Set $B:=A \otimes \delta^{-1}$ so that $A=B \otimes \delta$. Choose a Hermitian connection $\nabla^{B}$ of $B$. Denote by $\nabla^{k}$ the connection of $L^{k} \otimes B$ induced by $\nabla$ and $\nabla^{B}$. For any vector field $X$ of $M$, let $D_{X}^{K}$ be the derivative of $\mathcal{C}^{\infty}(M, K)$ in the direction of $X$ given by

$$
D_{X}^{K}(s)=p\left(\mathcal{L}_{X} s\right), \quad \forall s \in \mathcal{C}^{\infty}(M, K),
$$

where $p$ is the projection from $\wedge^{n}\left(T^{*} M \otimes \mathbb{C}\right)$ onto $K$ with kernel $\wedge^{n-1,1} T^{*} M \oplus$ $\ldots \oplus \wedge^{0, n} T^{*} M$. Introduce now the derivative $D_{X}^{\delta}$ of $\mathcal{C}^{\infty}(M, \delta)$ in the direction of $X$ such that

$$
D_{X}^{K}\left(\varphi\left(s^{2}\right)\right)=2 \varphi\left(s \otimes D_{X}^{\delta} s\right), \quad \forall s \in \mathcal{C}^{\infty}(M, \delta) .
$$

Finally for any $f \in \mathcal{C}^{\infty}(M)$, consider the operator

$$
Q_{k}(f)=\Pi_{k}\left(f+\frac{i}{k}\left(\nabla_{X}^{k} \otimes \mathrm{id}+\mathrm{id} \otimes D_{X}^{\delta}\right)\right): \mathcal{H}_{k} \rightarrow \mathcal{H}_{k}
$$

where $X$ is the Hamiltonian vector field of $f$, that is $d f=\omega(X, \cdot)$.

Theorem 1.1. For any Hermitian connection $\nabla^{B}$ of $B$, we have

1. For any $f \in \mathcal{C}^{\infty}(M), Q(f)=\left(Q_{k}(f)\right)$ is a Toeplitz operator with principal symbol $f$. 
2. For any $f$ and $g \in \mathcal{C}^{\infty}(M)$, we have

$$
Q_{k}(f) Q_{k}(g)=Q_{k}(f g)+\frac{1}{2 i k} Q_{k}(\{f, g\}) \quad \bmod k^{-2} \mathcal{T}
$$

and

$$
\left[Q_{k}(f), Q_{k}(g)\right]=\frac{1}{i k} Q_{k}(\{f, g\})+\frac{1}{i k^{2}} \Pi_{k}(R(X, Y)) \bmod k^{-3} \mathcal{T},
$$

where $X$ and $Y$ are the Hamiltonian vector fields of $f$ and $g$ respectively, $i R=\Theta(B) \in \Omega^{2}(M$, End $B)$ is the curvature of $\nabla^{B}$.

3. For any $f \in \mathcal{C}^{\infty}(M)$ and Toeplitz operator $S,\left(k i\left[Q_{k}(f), S\right]\right)$ is a Toeplitz operator with principal symbol

$$
\sigma_{\mathrm{p}}\left(k i\left[Q_{k}(f), S\right]\right)=-\nabla_{X}^{B}\left(\sigma_{\mathrm{p}}(S)\right) .
$$

where $X$ is the Hamiltonian vector field of $f$.

In this statement, we have identified End $A$ and End $B$ by using that $\delta$ is a line bundle so that $\operatorname{End}(\delta)$ is canonically isomorphic with the trivial line bundle. Furthermore, we let the covariant derivative $\nabla^{B}$ act on sections of End $B$ in the usual way. Observe that in the case where $B$ is a line bundle $\nabla_{X}^{B}\left(\sigma_{\mathrm{p}}(S)\right)=$ $X . \sigma_{\mathrm{p}}(S)$ so that Equation (4) becomes $\sigma_{\mathrm{p}}\left(k i\left[Q_{k}(f), S\right]\right)=\left\{f, \sigma_{\mathrm{p}}(S)\right\}$.

We can now defined a subprincipal symbol map $\sigma_{\mathrm{s}}: \mathcal{T}_{\mathrm{sc}} \rightarrow \mathcal{C}^{\infty}(M$, End $A)$ by $\sigma_{\mathrm{s}}(T):=\sigma_{\mathrm{p}}\left(k\left(T-Q\left(\sigma_{\mathrm{p}}(T)\right)\right)\right)$. In other words, for any Toeplitz operator $T \in \mathcal{T}_{\text {sc }}$, we have

$$
T=Q(f)+k^{-1} \Pi g \quad \bmod k^{-2} \mathcal{T}, \quad \text { with } f=\sigma_{\mathrm{p}}(T), g=\sigma_{\mathrm{s}}(T)
$$

By Theorem 1.1, $\sigma_{\mathrm{s}}$ satisfies i) ii) and iii) Furthermore, by (3) and (44), for any $S, T \in \mathcal{T}_{\mathrm{sc}}$,

$$
\sigma_{\mathrm{s}}(i k[T, S])=R(X, Y)-\nabla_{X}^{B} \sigma_{\mathrm{s}}(S)+\nabla_{Y}^{B} \sigma_{\mathrm{s}}(T)+i\left[\sigma_{\mathrm{s}}(T), \sigma_{\mathrm{s}}(S)\right]
$$

where $X$ and $Y$ are the Hamiltonian vector fields of $\sigma_{\mathrm{p}}(T)$ and $\sigma_{\mathrm{p}}(S)$ respectively.

Observe that $\sigma_{\mathrm{s}}$ is not uniquely defined, since it depends on the choice of $\nabla^{B}$. Actually, the space of Hermitian connections of $B$ is an affine space directed by $\Omega^{1}(M, \operatorname{Herm} B)$ where Herm $B$ is the bundle of Hermitian endomorphisms of $B$. As we will see, the space of subprincipal symbol maps is an affine space directed by $\mathcal{C}^{\infty}(M, T M \otimes \operatorname{Herm} A)$, the action being given by $\left(V+\sigma_{\mathrm{s}}\right)(T)=$ $\sigma_{\mathrm{s}}(T)+V \cdot \sigma_{\mathrm{p}}(T)$.

Proposition 1.2. The map from the space of connections of $B$ to the space of subprincipal symbol maps of $\mathcal{T}$, sending $\nabla^{B}$ to $\sigma_{\mathrm{s}}$ so that (5) is satisfied, is an isomorphism of affine vector spaces. Here the corresponding vector spaces $\Omega^{1}(M, \operatorname{Herm} B)$ and $\mathcal{C}^{\infty}(M, T M \otimes \operatorname{Herm} A)$ are identified through the symplectic duality $T^{*} M \simeq T M$ and the natural isomorphism $\operatorname{Herm} A \simeq \operatorname{Herm} B \otimes \operatorname{Herm} \delta \simeq$ Herm $B$ considered above. 
Remark 1.3. The case where $B$ is trivial, so that $\mathcal{H}_{k} \subset \mathcal{C}^{\infty}\left(M, L^{k} \otimes \delta\right)$, is usually called the quantization with metaplectic correction. There is a natural and very particular choice for the connection of $B: \nabla^{B}=d$. Then (3) simplifies

$$
\left[Q_{k}(f), Q_{k}(g)\right]=\frac{1}{i k} Q_{k}(\{f, g\}) \quad \bmod k^{-3} \mathcal{T}
$$

and the corresponding subprincipal symbol satisfies:

$$
\sigma_{\mathrm{s}}(i k[T, S])=\left\{\sigma_{\mathrm{p}}(T), \sigma_{\mathrm{s}}(S)\right\}+\left\{\sigma_{\mathrm{s}}(T), \sigma_{\mathrm{p}}(S)\right\} .
$$

This situation can be compared with the one of pseudo-differential operators acting on half-densities. In that case, there is a well-defined subprincipal symbol which satisfies (77). Let us note also that according to BdM02, only a map satisfying Equation (17) deserves the name of subprincipal symbol.

Remark 1.4. Equation (3) is relevant to classify the algebras $\mathcal{T}$ corresponding to various choices of $A$. For instance, assume that $A$ is a line bundle so that $R \in \Omega^{2}(M, \mathbb{R})$. Then the cohomology class of $R$ does not depend on the choice of the subprincipal symbol. Actually, $i R$ being the curvature of $\nabla^{B}$,

$$
\frac{1}{2 \pi}[R]=c_{1}(B)=c_{1}(A)+\frac{1}{2} c_{1}(M) .
$$

Assume now that we have another line bundle $A^{\prime}$ on $M$ with corresponding spaces $\mathcal{H}_{k}^{\prime} \subset \mathcal{C}^{\infty}\left(M, L^{k} \otimes A^{\prime}\right)$ and Toeplitz algebra $\mathcal{T}^{\prime}$. We may ask whether there exists a star-algebra morphism $\Phi: \mathcal{T} \rightarrow \mathcal{T}^{\prime}$ such that $\Phi\left(k^{-1} T\right)=k^{-1} \Phi(T)$ and $\sigma_{\mathrm{p}}(\Phi(T))=\sigma_{\mathrm{p}}(T)$ for any $T \in \mathcal{T}$. Observe that if $\Phi$ is such a morphism and $\sigma_{\mathrm{s}}^{\prime}$ is a subprincipal symbol map of $\mathcal{T}^{\prime}$, then $\sigma_{\mathrm{s}}:=\sigma_{\mathrm{s}}^{\prime} \circ \Phi$ is a subprincipal symbol map of $\mathcal{T}$. So as a consequence of Equation (3), a necessary condition for $\Phi$ to exist is that $A$ and $A^{\prime}$ have the same Chern class. In Cha07, we prove that this condition is also sufficient in the Kähler case. We plan to extend these results to the general symplectic case in a next paper.

Remark 1.5. Recall that the star products of $(M, \omega)$ are classified up to equivalence by their Fedosov class which is an element of $H^{2}(M, \mathbb{C})[[\hbar]]$, cf. Fed96. In the case where $A$ is a line bundle, the product $\star_{\text {cont }}$ of contravariant symbols of Toeplitz operators is a star product. By Equation (3), the first coefficient of the Fedosov class of $\star_{\text {cont }}$ is equal to $c_{1}(A)+\frac{1}{2} c_{1}(M)$.

Remark 1.6. In the Kähler case, there is a particular choice for the connection of $B$, namely the Chern connection. Doing this choice and assuming that $A$ is a line bundle, we recover the subprincipal symbol defined in equation (2), cf. Cha06. In this case, Equations (2) and (3) have been proved in Cha07. However, the proof in Cha07] was rather indirect and based on the morphisms which we alluded to in Remark 1.4. The proof we propose in this paper is much simpler.

Theorem 1.1 and Proposition 1.2 generalize to the case where there is no halfform bundle. The difficulty as we will see is to define the convenient operators 
$Q_{k}(f)$ in this case and to understand what replaces the choice of the connection on $B$.

The paper is organized as follows. In Section 22 we recall some basic facts on Toeplitz operators. In Section 3 we start the study of subprincipal symbols and we go as far as possible without using the operators $Q_{k}(f)$. We will see that we can deduce Equation (6) from almost nothing, but without computing explicitly $R$ and $\nabla^{B}$. In section 4 we introduce some material related to halfform bundles. In section 5 , we define the operator $Q_{k}(f)$ and state the theorem generalizing Theorem 1.1 in the absence of half-form bundle. Sections 6 and 7 are devoted to the proof of this result.

\section{Toeplitz operators}

Consider a compact symplectic manifold $M$ equipped with a prequantum bundle $L \rightarrow M$. Recall that $L$ is a Hermitian line bundle with a connection of curvature $\frac{1}{i} \omega$. Let $A$ be any Hermitian vector bundle. Let $j$ be an almost complex structure of $M$ compatible with $\omega$. Consider a family

$$
\mathcal{H}=\left(\mathcal{H}_{k} \subset \mathcal{C}^{\infty}\left(M, A \otimes L^{k}\right), k \in \mathbb{N}\right)
$$

of finite dimensional subspaces. Assume that the orthogonal projector $\Pi_{k}$ onto $\mathcal{H}_{k}$ belongs to the algebra $\mathcal{A}_{0}$ introduced in Section 6 .

A Toeplitz operator is any family $\left(T_{k}: \mathcal{H}_{k} \rightarrow \mathcal{H}_{k}, k \in \mathbb{N}\right)$ of operators of the form

$$
T_{k}=\Pi_{k} f(\cdot, k)+R_{k}, \quad k \in \mathbb{N}^{*}
$$

where $f(\cdot, k)$, viewed as a multiplication operator, is a sequence in $\mathcal{C}^{\infty}(M$, End $A)$ admitting an asymptotic expansion $f_{0}+k^{-1} f_{1}+\ldots$ for the $\mathcal{C}^{\infty}$ topology. Furthermore the norm of $R_{k} \in$ End $\mathcal{H}_{k}$ is in $\mathcal{O}\left(k^{-\infty}\right)=\bigcap_{N} \mathcal{O}\left(k^{-N}\right)$.

The following facts are proved in Cha15. The space $\mathcal{T}$ of Toeplitz operators is a star-algebra with identity $\left(\Pi_{k}\right)$, the product being the usual composition of operators, the involution being the Hermitian adjoint. The symbol map

$$
\sigma_{\text {cont }}: \mathcal{T} \rightarrow \mathcal{C}^{\infty}(M, \text { End } A)[[\hbar]]
$$

sending $\left(T_{k}\right)$ into the formal series $f_{0}+\hbar f_{1}+\ldots$ where the functions $f_{i}$ are the coefficients of the asymptotic expansion of the multiplicator $f(\cdot, k)$ is well defined. It is onto and its kernel is the ideal consisting of $\mathcal{O}\left(k^{-\infty}\right)$ Toeplitz operators. More precisely, for any integer $\ell,\left\|T_{k}\right\|=\mathcal{O}\left(k^{-\ell}\right)$ if and only if $\sigma_{\text {cont }}(T)=\mathcal{O}\left(\hbar^{\ell}\right)$. According to Berezin terminology, $\sigma_{\text {cont }}(T)$ is called the contravariant symbol of $T$.

The principal symbol $\sigma_{\mathrm{p}}(T) \in \mathcal{C}^{\infty}(M$, End $A)$ is by definition the first coefficient of the contravariant symbol, so $\sigma_{\text {cont }}(T)=\sigma_{\mathrm{p}}(T)+\mathcal{O}(\hbar)$. The principal symbol map

$$
\sigma_{\mathrm{p}}: \mathcal{T} \rightarrow \mathcal{C}^{\infty}(M, \text { End } A)
$$


is onto and $\sigma_{\mathrm{p}}(T)=0$ if and only if there exists $S \in \mathcal{T}$ such that $T=k^{-1} S$. For any Toeplitz operators $T, S \in \mathcal{T}$ with principal symbols $f$ and $g$, we have $\sigma_{\mathrm{p}}(T S)=f . g$. If $f$ and $g$ are scalar valued, then $i k[T, S] \in \mathcal{T}$ and

$$
\sigma_{\mathrm{p}}(i k[T, S])=\{f, g\},
$$

where $\{\cdot, \cdot\}$ is the Poisson bracket of $M$. Denoting by $\left\|T_{k}\right\|$ the operator norm of $T_{k}$ corresponding to the scalar product of $\mathcal{H}_{k} \subset \mathcal{C}^{\infty}\left(M, L^{k} \otimes A\right)$, we have

$$
\left\|T_{k}\right\|=\sup _{y \in M}\left|\sigma_{\mathrm{p}}(T)(y)\right|+\mathcal{O}\left(k^{-1}\right)
$$

where for any $y \in M,\left|\sigma_{\mathrm{p}}(T)(y)\right|$ is the operator norm of $\sigma_{\mathrm{p}}(T)(y) \in$ End $A_{y}$. Consequently, $\sigma_{\mathrm{p}}(T)=0$ if and only if $\left\|T_{k}\right\|=\mathcal{O}\left(k^{-1}\right)$.

As a last property, the full product of contravariant symbols has the following form: if $\sigma_{\text {cont }}(T)=\sum \hbar^{\ell} f_{\ell}$ and $\sigma_{\text {cont }}(S)=\sum \hbar^{\ell} g_{\ell}$, then

$$
\sigma_{\text {cont }}(T S)=\sum_{\ell} \hbar^{\ell} \sum_{\ell=p+q+r} B_{r}\left(f_{p}, g_{q}\right)
$$

where $B_{0}(f, g)=f g$ and for any $r, B_{r}: \mathcal{C}^{\infty}(M$, End $A) \times \mathcal{C}^{\infty}(M$, End $A) \rightarrow$ $\mathcal{C}^{\infty}(M$, End $A)$ is a bilinear local operator, cf. Cha15].

\section{Subprincipal symbols}

Denote by $\mathcal{T}_{\text {sc }}$ the set of Toeplitz operator $T \in \mathcal{T}$ with a scalar principal symbol. Let $\mathcal{E}$ be the set of linear map $\sigma_{\mathrm{s}}: \mathcal{T}_{\mathrm{sc}} \rightarrow \mathcal{C}^{\infty}(M$, End $A)$ satisfying the following conditions:

i) for any $T \in \mathcal{T}, \sigma_{\mathrm{s}}\left(k^{-1} T\right)=\sigma_{\mathrm{p}}(T)$,

ii) for any $T \in \mathcal{T}_{\mathrm{sc}}, \sigma_{\mathrm{s}}\left(T^{*}\right)=\sigma_{\mathrm{s}}(T)^{*}$,

iii) for any $T, S \in \mathcal{T}_{\mathrm{sc}}, \sigma_{\mathrm{s}}(T S)=\sigma_{\mathrm{p}}(T) \sigma_{\mathrm{s}}(S)+\sigma_{\mathrm{s}}(T) \sigma_{\mathrm{p}}(S)+\frac{1}{2 i}\left\{\sigma_{\mathrm{p}}(T), \sigma_{\mathrm{p}}(S)\right\}$,

We will first prove that $\mathcal{E}$ is not empty. Define the contravariant subprincipal symbol map $\sigma_{\mathrm{s}}^{\mathrm{c}}: \mathcal{T}_{\mathrm{sc}} \rightarrow \mathcal{C}^{\infty}(M$, End $A)$ as follows:

$$
\sigma_{\text {cont }}(T)=\sigma_{\mathrm{p}}(T)+\hbar \sigma_{\mathrm{s}}^{\mathrm{c}}(T)+\mathcal{O}\left(\hbar^{2}\right)
$$

Then $\sigma_{\mathrm{s}}^{\mathrm{c}}$ satisfies i) ii) but not iii). Actually, by (10), we have

$$
\sigma_{\mathrm{s}}^{\mathrm{c}}(T S)=\sigma_{\mathrm{p}}(T) \sigma_{\mathrm{s}}^{\mathrm{c}}(S)+\sigma_{\mathrm{s}}^{\mathrm{c}}(T) \sigma_{\mathrm{p}}(S)+B\left(\sigma_{\mathrm{p}}(T), \sigma_{\mathrm{p}}(S)\right)
$$

where $B$ is a bidifferential operator from $\mathcal{C}^{\infty}(M) \times \mathcal{C}^{\infty}(M)$ to $\mathcal{C}^{\infty}(M$, End $A)$. By (9), the antisymmetric part of $B$ is equal to $\frac{1}{2 i}$ times the Poisson bracket, so

$$
B(f, g)=B^{\mathrm{s}}(f, g)+\frac{1}{2 i}\{f, g\}
$$

where $B^{\mathrm{s}}$ is symmetric. We will modify $\sigma_{\mathrm{s}}^{\mathrm{c}}$ to get a subprincipal symbol satisfying iii), The method we follow is based on standard lemmas in Deformation quantization. 
Proposition 3.1. Let $B^{\mathrm{s}}: \mathcal{C}^{\infty}(M) \times \mathcal{C}^{\infty}(M) \rightarrow \mathcal{C}^{\infty}(M$, End $A)$ be any bidifferential symmetric operator satisfying

$$
B^{\mathrm{s}}(f, g) h+B^{\mathrm{s}}(f g, h)=B^{\mathrm{s}}(f, g h)+f B^{\mathrm{s}}(g, h), \quad \forall f, g, h \in \mathcal{C}^{\infty}(M) .
$$

Then there exists a differential operator $Q: \mathcal{C}^{\infty}(M) \rightarrow \mathcal{C}^{\infty}(M$, End $A)$ such that for any $f, g \in \mathcal{C}^{\infty}(M)$, we have $B^{\mathrm{s}}(f, g)=f Q(g)+Q(f) g-Q(f g)$.

In the case where $A$ is the trivial line bundle over $M$, this result is a classical lemma in Hochschild cohomology saying that any cocycle with a null antisymmetric part is exact, cf. [BCG97] for a short proof. It is easy to deduce Proposition 3.1 from this particular case.

Using that the product of Toeplitz operators is associative, a straightforward computations shows that the bidifferential operator $B$ defined by (12) satisfies the equality $B(f, g) h+B(f g, h)=B(f, g h)+f B(g, h)$. Using that the Poisson bracket is a derivative with respect to each of his arguments, we conclude that the symmetric part $B^{\mathrm{s}}$ of $B$ satisfies (14). Applying Proposition 3.1, we get a differential operator $Q$. Then a straightforward computation shows that $\sigma_{\mathrm{s}}:=$ $\sigma_{\mathrm{s}}^{\mathrm{c}}+Q \circ \sigma_{\mathrm{p}}$ satisfies iii). Since $\sigma_{\mathrm{s}}^{\mathrm{c}}$ satisfies ii) $B^{\mathrm{s}}$ is real in the sense that $B^{\mathrm{s}}$ is the complexification of a $\mathbb{R}$-bilinear map $\mathcal{C}^{\infty}(M, \mathbb{R}) \times \mathcal{C}^{\infty}(M, \mathbb{R}) \rightarrow \mathcal{C}^{\infty}(M$, Herm $A)$, where Herm $A \rightarrow M$ is the vector bundle of Hermitian endomorphisms of $A$. $B^{\mathrm{s}}$ being real, we can choose $Q$ real, so that $\sigma_{\mathrm{s}}^{\mathrm{c}}$ satisfies ii) as well. We have proved that $\mathcal{E}$ is not empty.

Now consider $\sigma_{\mathrm{s}} \in \mathcal{E}$ and $V \in \mathcal{C}^{\infty}(M, T M \otimes \operatorname{Herm} A)$. Then the map $\sigma_{\mathrm{s}}^{\prime}$ defined by

$$
\sigma_{\mathrm{s}}^{\prime}(T):=\sigma_{\mathrm{s}}(T)+d f(V) \quad \text { where } \quad f=\sigma_{\mathrm{p}}(T),
$$

satisfies i), ii) [iii). Conversely, let $\sigma_{\mathrm{s}}$ and $\sigma_{\mathrm{s}}^{\prime}$ in $\mathcal{E}$. By i), $\sigma_{\mathrm{s}}^{\prime}-\sigma_{\mathrm{s}}$ vanishes on $k^{-1} \mathcal{T}$. Since $k^{-1} \mathcal{T}$ is the kernel of the principal symbol map, we have $\sigma_{\mathrm{s}}^{\prime}(T)-\sigma_{\mathrm{s}}(T)=D\left(\sigma_{p}(T)\right)$ where $D: \mathcal{C}^{\infty}(M) \rightarrow \mathcal{C}^{\infty}(M$, End $A)$. By ii) $D$ is real. By iii), $D$ is a derivation, that is $D(f g)=f D(g)+g D(f)$. We conclude that $D(f)=d f(V)$ for some $V \in \mathcal{C}^{\infty}(M, T M \otimes \operatorname{Herm} A)$. To summarize we have proved the following Proposition.

Proposition 3.2. $\mathcal{E}$ is an affine space with associated vector space $\mathcal{C}^{\infty}(M, T M \otimes$ $\operatorname{Herm} A)$.

It is helpful to view $\sigma_{\mathrm{s}} \in \mathcal{E}$ as a first order deformation of the principal symbol. To give a sense to this, denote by $\mathcal{S}$ be the vector space $\mathcal{C}^{\infty}(M) \oplus$ $\hbar \mathcal{C}^{\infty}(M$, End $A)$ and define the map $\sigma: \mathcal{T}_{\text {sc }} \rightarrow \mathcal{S}$ by

$$
\sigma(T)=\sigma_{\mathrm{p}}(T)+\hbar \sigma_{\mathrm{s}}(T) .
$$

Using that $\sigma_{\mathrm{p}}$ is onto with kernel $k^{-1} \mathcal{T}$ and that $\sigma_{\mathrm{s}}$ satisfies i) we see that $\sigma$ is onto with kernel $k^{-2} \mathcal{T}$. By ii), $\sigma$ is real. Furthermore by iii), $\sigma(S T)=$ $\sigma(S) \star \sigma(T)$ where $\star$ is the product of $\mathcal{S}$ given by

$$
\left(f_{0}+\hbar f_{1}\right) \star\left(g_{0}+\hbar g_{1}\right)=f_{0} g_{0}+\hbar\left(f_{0} g_{1}+f_{1} g_{0}+\frac{1}{2 i}\left\{f_{0}, g_{0}\right\}\right) .
$$


1 being the identity of $\star$, we easily get that $\sigma(\mathrm{id})=1$.

For any $T, S \in \mathcal{T}_{\text {sc }}, k[T, S]$ is a Toeplitz operator with scalar symbol. Furthermore observe that the class of $k[T, S]$ modulo $\mathcal{O}\left(k^{-2}\right)$ only depend on the classes of $T$ and $S$ modulo $\mathcal{O}\left(k^{-2}\right)$. So there exists a unique bilinear map

$$
[\cdot, \cdot]_{\sigma}: \mathcal{S} \times \mathcal{S} \rightarrow \mathcal{S}
$$

such that $\sigma(i k[T, S])=[\sigma(T), \sigma(S)]_{\sigma}$ for any $T, S \in \mathcal{T}_{\mathrm{sc}}$. Since the commutator of endomorphisms is a derivation with respect to each argument and satisfies the Jacobi identity, we obtain that for any $f, g, h \in \mathcal{S}$

$$
[f \star g, h]_{\sigma}=f \star[g, h]_{\sigma}+[f, h]_{\sigma} \star g .
$$

and

$$
\left[f,[g, h]_{\sigma}\right]_{\sigma}+\left[g,[h, f]_{\sigma}\right]_{\sigma}+\left[h,[f, g]_{\sigma}\right]_{\sigma}=0
$$

Furthermore, $[f, g]_{\sigma}=-[g, f]_{\sigma}$ and $\left[f^{*}, g^{*}\right]_{\sigma}=[f, g]_{\sigma}^{*}$. Exploiting these equations, we deduce the following proposition.

Proposition 3.3. For any $\sigma_{\mathrm{s}} \in \mathcal{E}$, there exists $R \in \Omega^{2}(M$, Herm $A)$ and a connection $\nabla: \mathcal{C}^{\infty}(M, \operatorname{Herm} A) \rightarrow \Omega^{1}(M, \operatorname{Herm} A)$ such that for any $f_{0}+\hbar f_{1}$, $g_{0}+\hbar g_{1} \in \mathcal{S}$ we have

$$
\left[f_{0}+\hbar f_{1}, g_{0}+\hbar g_{1}\right]_{\sigma}=\left\{f_{0}, g_{0}\right\}+\hbar\left(R(X, Y)-\nabla_{X} g_{1}+\nabla_{Y} f_{1}+i\left[f_{1}, g_{1}\right]\right)
$$

where $X$ and $Y$ are the Hamiltonian vector fields of $f_{0}$ and $g_{0}$. Furthermore,

$$
\operatorname{courb} \nabla=i \operatorname{ad}_{R}, \quad \nabla R=0, \quad \nabla \mathrm{id}=0
$$

and $\nabla\left(f_{1} . g_{1}\right)=\left(\nabla f_{1}\right) \cdot g_{1}+f_{1} \cdot\left(\nabla g_{1}\right)$ for any $f_{1}, g_{1} \in \mathcal{C}^{\infty}(M$, Herm $A)$.

Proof. By condition i) and the properties of the principal symbol, we have

$\left[f_{0}+\hbar f_{1}, g_{0}+\hbar g_{1}\right]_{\sigma}=\left\{f_{0}, g_{0}\right\}+\hbar\left(A\left(f_{0}, g_{0}\right)+B\left(f_{0}, g_{1}\right)+C\left(g_{0}, f_{1}\right)+i\left[f_{1}, g_{1}\right]\right)$,

where $A, B, C$ are bilinear operators with value in $\mathcal{C}^{\infty}(M$, End $A), A$ being defined on $\mathcal{C}^{\infty}(M) \times \mathcal{C}^{\infty}(M)$ and $B, C$ on $\mathcal{C}^{\infty}(M) \times \mathcal{C}^{\infty}(M$, End $A)$. Since $[\cdot, \cdot]_{\sigma}$ is antisymmetric, we have that

$$
A\left(f_{0}, g_{0}\right)=-A\left(g_{0}, f_{0}\right), \quad B\left(f_{0}, g_{1}\right)=-C\left(f_{0}, g_{1}\right)
$$

Since $\sigma$ is real and $\sigma(\mathrm{id})=1,[\cdot, \cdot]_{\sigma}$ is real and $[1, \cdot]_{\sigma}=0$. Consequently, $A$ and $B$ are real, meaning that $A\left(\bar{f}_{0}, \bar{g}_{0}\right)=A\left(f_{0}, g_{0}\right)^{*}$ and $B\left(\bar{f}_{0}, g_{1}^{*}\right)=B\left(f_{0}, g_{1}\right)^{*}$. Furthermore

$$
A\left(1, g_{0}\right)=0, \quad B\left(1, g_{1}\right)=0, \quad B\left(f_{0}, \mathrm{id}\right)=0 .
$$

The last equation follows from the fact that $\sigma\left(k^{-1} \mathrm{id}\right)=\hbar \mathrm{id}$ so that $[\hbar \mathrm{id}, \cdot]_{\sigma}=0$. By equation (15), for any $f_{0}, g_{0} \in \mathcal{C}^{\infty}(M)$, and $h_{1} \in \mathcal{C}^{\infty}(M$, End $A)$

$$
\left[f_{0} \star g_{0}, \hbar h_{1}\right]_{\sigma}=f_{0} \star\left[g_{0}, \hbar h_{1}\right]_{\sigma}+\left[f_{0}, \hbar h_{1}\right]_{\sigma} \star g_{0}
$$


so that

$$
B\left(f_{0} g_{0}, h_{1}\right)=f_{0} B\left(g_{0}, h_{1}\right)+g_{0} B\left(f_{0}, h_{1}\right)
$$

Furthermore, as another application of Equation (15),

$$
\left[f_{0}, g_{0} \star \hbar h_{1}\right]_{\sigma}=\left[f_{0}, g_{0}\right]_{\sigma} \star \hbar h_{1}+g_{0} \star\left[f_{0}, \hbar h_{1}\right]_{\sigma}
$$

so that

$$
B\left(f_{0}, g_{0} h_{1}\right)=\left\{f_{0}, g_{0}\right\} h_{1}+g_{0} B\left(f_{0}, h_{1}\right)=-\left(X . g_{0}\right) h_{1}+g_{0} B\left(f_{0}, h_{1}\right) .
$$

where $X$ is the Hamiltonian vector field of $f_{0}$. By Equation (20), $B\left(f_{0}, \cdot\right)$ is a derivative of $\mathcal{C}^{\infty}(M$, End $A)$ in the direction of $-X$. By Equation (19) and the second equation of (18), for any $p \in M, B\left(f_{0}, g_{1}\right)(p)=B\left(f_{0}^{\prime}, g_{1}\right)(p)$ if $f_{0}$ and $f_{0}^{\prime}$ have the same differential at $p$. These two facts imply that $B\left(f_{0}, h_{1}\right)=-\nabla_{X} h_{1}$ for a connection $\nabla: \mathcal{C}^{\infty}(M$, End $A) \rightarrow \Omega^{1}(M$, End $A)$. Since $B$ is real, $\nabla$ is actually the complexification of a connection $\mathcal{C}^{\infty}(M, \operatorname{Herm} A) \rightarrow \Omega^{1}(M, \operatorname{Herm} A)$. Furthermore by the last equation of (18), $\nabla$ id $=0$.

Consider now $f, g, h \in \mathcal{C}^{\infty}(M)$. Expanding Equation (15) and using Jacobi identity for the Poisson bracket, we obtain that

$$
A(f g, h)=f A(g, h)+g A(f, h) .
$$

Since furthermore $A$ is antisymmetric and vanishes on the constant, we conclude that $A(f, g)=R(X, Y)$ with $R \in \Omega^{2}(M$, End $A)$ and $X, Y$ the Hamiltonian vector fields of $f$ and $g$. Since $A$ is real, $R \in \Omega^{2}(M, \operatorname{Herm} A)$.

Still working with $f, g, h \in \mathcal{C}^{\infty}(M)$ and denoting by $X, Y$ and $Z$ their Hamiltonian vector fields, we have

$$
\begin{aligned}
{\left[f,[g, h]_{\sigma}\right]_{\sigma} } & =\{f,\{g, h\}\}+\hbar(A(f,\{g, h\})+B(f, A(g, h))) \\
& =\{f,\{g, h\}\}-\hbar\left(R(X,[Y, Z])+\nabla_{X} R(Y, Z)\right)
\end{aligned}
$$

Since the bracket $[\cdot, \cdot]_{\sigma}$ satisfies the Jacobi identity, this shows that $(\nabla R)(X, Y, Z)=$ 0 so that $\nabla R=0$. Another application of the Jacobi identity (16) with $f, g \in \mathcal{C}^{\infty}(M)$ and $h \in \hbar \mathcal{C}^{\infty}(M$, End $A)$ leads to

$$
\left[\nabla_{X}, \nabla_{Y}\right] h_{1}-\nabla_{[X, Y]} h_{1}=i\left[R(X, Y), h_{1}\right]
$$

which means that the curvature of $\nabla$ is $i \operatorname{ad}_{R}$. The last equation to prove does not follows from the previous conditions. Consider three Toeplitz operators $R$, $S$ and $T$ with principal symbols $f_{0} \in \mathcal{C}^{\infty}(M), g_{1}, h_{1} \in \mathcal{C}^{\infty}(M$, End $A)$. Starting from $i k\left[T, k^{-1} R S\right]=R\left(i k\left[T, k^{-1} S\right]\right)+\left(i k\left[T, k^{-1} R\right]\right) S$, we obtain that

$$
\nabla_{X}\left(g_{1} \cdot h_{1}\right)=g_{1} \cdot\left(\nabla_{X} h_{1}\right)+\left(\nabla_{X} g_{1}\right) \cdot h_{1}
$$

where $X$ is the Hamiltonian vector field of $f_{0}$.

We can also easily compute how the form $R$ and the connection $\nabla$ are changed when we choose a new subprincipal symbol. 
Lemma 3.4. Let $\sigma_{\mathrm{s}} \in \mathcal{E}, V \in \mathcal{C}^{\infty}(M, T M \otimes \operatorname{Herm} A)$ and set $\sigma_{\mathrm{s}}^{\prime}=\sigma_{\mathrm{s}}+V . \sigma_{\mathrm{p}}$. Then the connections $\nabla, \nabla^{\prime}$ and the two forms $R, R^{\prime}$ corresponding respectively to $\sigma_{\mathrm{s}}$ and $\sigma_{\mathrm{s}}^{\prime}$ satisfy

$$
\nabla^{\prime}=\nabla+i \operatorname{ad}_{\alpha}, \quad R^{\prime}=R+\nabla \alpha+i \alpha \wedge \alpha
$$

where $\alpha \in \Omega^{1}(M, \operatorname{Herm} A)$ is given by $\alpha=\omega(V, \cdot)$.

The case where $A$ is a line bundle is already interesting. By the following corollary, the cohomology class $[R] \in H^{2}(M, \mathbb{R})$ does not depend on the choice of the subprincipal symbol map.

Corollary 3.5. Assume that $A$ is a line bundle. Then for any $\sigma_{\mathrm{s}} \in \mathcal{E}$, we have

$$
\left[f_{0}+\hbar f_{1}, g_{0}+\hbar g_{1}\right]_{\sigma}=\left\{f_{0}, g_{0}\right\}+\hbar\left(R(X, Y)-\mathcal{L}_{X} g_{1}+\mathcal{L}_{Y} f_{1}\right)
$$

where $R \in \Omega^{2}(M, \mathbb{R})$ is closed and $\mathcal{L}_{X}, \mathcal{L}_{Y}$ are the Lie derivatives with respect to the Hamiltonian vector fields of $f_{0}$ and $g_{0}$ respectively. Furthermore, the twoform $R^{\prime}$ corresponding to $\sigma_{\mathrm{s}}^{\prime}=\sigma_{\mathrm{s}}+V \cdot \sigma_{\mathrm{p}}$ is given by $R^{\prime}=R+d \iota_{V} \omega$. So the cohomology class of $R$ does not depend on the choice of $\sigma_{\mathrm{s}}$.

Proof. Since $A$ is a line bundle, Herm $A$ is naturally isomorphic with the trivial real line bundle. The fact that $\nabla$ id $=0$ implies that $\nabla$ is the de Rham derivation.

At this point, we could believe that for any $\sigma_{\mathrm{s}} \in \mathcal{E}$, there exists some connection $\nabla^{A}: \mathcal{C}^{\infty}(M, A) \rightarrow \Omega^{1}(M, A)$ preserving the metric of $A$, with curvature $R$ and such that $\nabla$ is the corresponding connection of Herm $A$. Indeed this would explain the equations in Proposition 3.3 We could also believe that the connection corresponding to $\sigma_{\mathrm{s}}^{\prime}=\sigma_{\mathrm{s}}+V \cdot \sigma_{\mathrm{p}}$ is given by $\nabla^{A}+\frac{1}{i} \iota_{V} \omega$ which would imply the equations in Lemma 3.4.

As we will see, this explanation almost holds, but we have to take into account the metaplectic correction. For instance, we will see that in the case where $A$ is a line bundle, the cohomology class of $\left[\frac{1}{2 \pi} R\right]$ is not $c_{1}(A)$ but $c_{1}(A)+$ $\frac{1}{2} c_{1}(M)$.

\section{Half-form computations}

\subsection{Canonical bundle and derivatives}

Let $2 n$ be the dimension of $M$. Let $K=\wedge^{n, 0} T^{*} M$ be the canonical bundle of $M$ with respect to $j$. Denote by $p$ the projection $\wedge^{n}\left(T^{*} M \otimes \mathbb{C}\right) \rightarrow \wedge^{n, 0} T^{*} M$ with kernel $\wedge^{n-1,1} T^{*} M \oplus \ldots \oplus \wedge^{0, n} T^{*} M$. Then for any vector field $X$ of $M$, introduce the derivative

$$
D_{X}^{K}: \mathcal{C}^{\infty}(M, K) \rightarrow \mathcal{C}^{\infty}(M, K), \quad D_{X}^{K} \mu=p \mathcal{L}_{X} \mu
$$

where $\mathcal{L}_{X}$ is the Lie derivative with respect to $X$. 
Lemma 4.1. For any $X \in \mathcal{C}^{\infty}(M, T M)$ and $f \in \mathcal{C}^{\infty}(M)$ we have

$$
D_{f X}^{K}=f D_{X}^{K}+d f\left(X^{1,0}\right)
$$

where $X^{1,0} \in \mathcal{C}^{\infty}\left(M, T^{1,0} M\right)$ is the $(1,0)$-component of $X$. Furthermore if $X$ is symplectic, $D_{X}^{K}$ preserves the metric of $K$ induced by $\omega$.

$D_{X}^{K}$ preserves the metric means that for any sections $s, t \in \mathcal{C}^{\infty}(M, K)$ we have

$$
\mathcal{L}_{X}(s, t)=\left(D_{X}^{K} s, t\right)+\left(s, D_{X}^{K} t\right)
$$

where $(s, t)$ is the pointwise scalar product. Equivalently we say that $D_{X}^{K}$ is Hermitian.

Proof. By Cartan formula, we have $\mathcal{L}_{f X}-f \mathcal{L}_{X}=d f \wedge \iota_{X}$. Introduce a local frame $\left(\partial_{i}\right)$ of $T^{1,0} M$ and denote by $\left(\theta_{i}\right)$ the dual frame. Since $D_{f X}^{K}$ and $f D_{X}^{K}$ are both derivatives in the direction of $f X$, they differ by the multiplication by a function. We compute this function by testing on the frame $\theta=\theta_{1} \wedge \ldots \wedge \theta_{n}$ of the canonical bundle. Write $X^{1,0}=\sum X_{i} \partial_{i}$ so that

$$
\iota_{X} \theta=\sum(-1)^{i+1} X_{i} \theta_{1} \wedge \ldots \wedge \hat{\theta}_{i} \wedge \ldots \wedge \theta_{n}
$$

and

$$
p\left(d f \wedge \iota_{X} \theta\right)=\sum X_{i} d f\left(\partial_{i}\right) \theta=d f\left(X^{1,0}\right) \theta .
$$

Consequently, $D_{f X}^{K}=f D_{X}^{K}+d f\left(X^{1,0}\right)$.

Let us prove that $D_{X}^{K}$ preserves the metric of $K$ when $X$ is symplectic. Recall that for any sections $s, t$ of $K, s \wedge \bar{t}=C_{n}(s, t) \omega^{n}$ for some constant $C_{n}$ independent of $s, t$. Since

$$
\mathcal{L}_{X}(s \wedge \bar{t})=\left(\mathcal{L}_{X} s\right) \wedge \bar{t}+s \wedge \overline{\mathcal{L}_{X} t}=\left(D_{X}^{K} s\right) \wedge \bar{t}+s \wedge \overline{D_{X}^{K} t},
$$

we deduce that $\mathcal{L}_{X}(s, t)=\left(D_{X}^{K} s, t\right)+\left(s, D_{X}^{K} t\right)$ when $X$ satisfies $\mathcal{L}_{X} \omega^{n}=0$.

Lemma 4.2. For any vector fields $X, Y$ of $M$, we have

$$
\left[D_{X}^{K}, D_{Y}^{K}\right]=D_{[X, Y]}^{K}+B_{j}(X, Y)
$$

where $B_{j}(X, Y)$ is the function of $M$ given by

$$
B_{j}(X, Y)=\sum\left(\mathcal{L}_{X} \theta_{i}\right)\left(\bar{\partial}_{j}\right)\left(\mathcal{L}_{Y} \bar{\theta}_{j}\right)\left(\partial_{i}\right)-\left(\mathcal{L}_{Y} \theta_{i}\right)\left(\bar{\partial}_{j}\right)\left(\mathcal{L}_{X} \bar{\theta}_{j}\right)\left(\partial_{i}\right)
$$

with $\left(\partial_{i}\right)$ a local frame of $T^{1,0} M$ and $\left(\theta_{i}\right)$ the dual frame.

Proof. Since

$$
p \mathcal{L}_{X} p \mathcal{L}_{Y} p-p \mathcal{L}_{X} \mathcal{L}_{Y} p=p \mathcal{L}_{X}(p-\mathrm{id}) \mathcal{L}_{Y} p,
$$

we have

$$
\left[D_{X}^{K}, D_{Y}^{K}\right]-D_{[X, Y]}^{K}=p \mathcal{L}_{X}(p-\mathrm{id}) \mathcal{L}_{Y}-p \mathcal{L}_{Y}(p-\mathrm{id}) \mathcal{L}_{X}
$$


Let $\theta=\theta_{1} \wedge \ldots \wedge \theta_{n}$. We have

$$
\mathcal{L}_{Y} \theta=\sum(-1)^{i+1}\left(\mathcal{L}_{Y} \theta_{i}\right) \wedge \theta_{1} \wedge \ldots \wedge \hat{\theta}_{i} \wedge \ldots \wedge \theta_{n}
$$

so that

$$
(p-\mathrm{id}) \mathcal{L}_{Y} \theta=\sum(-1)^{i}\left(\mathcal{L}_{Y} \theta_{i}\right)\left(\bar{\partial}_{j}\right) \bar{\theta}_{j} \wedge \theta_{1} \wedge \ldots \wedge \hat{\theta}_{i} \wedge \ldots \wedge \theta_{n}
$$

and

$$
p \mathcal{L}_{X}(p-\mathrm{id}) \mathcal{L}_{Y} \theta=-\sum\left(\mathcal{L}_{Y} \theta_{i}\right)\left(\bar{\partial}_{j}\right)\left(\mathcal{L}_{X} \bar{\theta}_{j}\right)\left(\partial_{i}\right) \theta
$$

The final result follows.

\subsection{Metaplectic correction}

Consider the set $\mathcal{D}$ of linear map $D: \mathcal{C}^{\infty}(M, T M) \rightarrow \operatorname{End}\left(\mathcal{C}^{\infty}(M, A)\right)$ satisfying the following conditions

$$
\begin{gathered}
D_{X}(f s)=(X . f) s+f D_{X} s \\
D_{f X} s=f D_{X} s+\frac{1}{2} d f\left(X^{1,0}\right) s
\end{gathered}
$$

for any vector field $Y$ of $M$, function $f \in \mathcal{C}^{\infty}(M)$ and sections $s, t \in \mathcal{C}^{\infty}(M, A)$. In the case where $X$ is a symplectic vector field, we also require that $D_{X}$ is Hermitian,

$$
X .(s, t)=\left(D_{X} s, t\right)+\left(s, D_{X} t\right), \quad s, t \in \mathcal{C}^{\infty}(M, A)
$$

where $(s, t) \in \mathcal{C}^{\infty}(M)$ is the pointwise scalar product of $s$ and $t$. Observe that for any $D \in \mathcal{D}$ and $\alpha \in \Omega^{1}(M, \operatorname{Herm} A), D+\frac{1}{i} \alpha$ belongs to $\mathcal{D}$.

Proposition 4.3. The space $\mathcal{D}$ is a real affine space directed by $\Omega^{1}(M, \operatorname{Herm} A)$.

Proof. The only difficulty is to check that $\mathcal{D}$ is not empty. To do that, we will use the derivations $D_{X}^{K}$ introduced in (21). Let $\nabla^{A}$ and $\nabla^{K}$ be Hermitian connections of $A$ and $K$ respectively. For any vector field $X$, define

$$
D_{X}:=\nabla_{X}^{A}+\frac{1}{2} B(X) \operatorname{id}_{A}: \mathcal{C}^{\infty}(M, A) \rightarrow \mathcal{C}^{\infty}(M, A)
$$

where $B(X) \in \mathcal{C}^{\infty}(M)$ is given by $B(X)=D_{X}^{K}-\nabla_{X}^{K} . D_{X}$ is clearly a derivation in the direction of $X$. By Lemma 4.1, $D_{X}$ satisfies Condition (23) and preserves the metric when $X$ is symplectic.

Remark 4.4. Let $\delta$ be a half-form bundle, that is a Hermitian line bundle such that $\delta^{2}$ is isomorphic to $K$. For any vector field $X$ of $M$, let $D_{X}^{\delta}$ be the derivative of $\mathcal{C}^{\infty}(M, \delta)$ in the direction of $X$ such that

$$
D_{X}^{K} s^{2}=2 s \otimes D_{X}^{\delta} s, \quad \forall s \in \mathcal{C}^{\infty}(M, \delta) .
$$


Write $A=B \otimes \delta$ where $B=A \otimes \delta^{-1}$. Then there is a one to one correspondence between the space of Hermitian connections of $B$ and $\mathcal{D}$ given as follows: for any Hermitian connection $\nabla^{B}$ of $B$, we set

$$
D_{Y}=\nabla_{Y}^{B} \otimes \mathrm{id}+\mathrm{id} \otimes D_{Y}^{\delta} .
$$

This provides another proof of Proposition 4.1 in the case where $M$ has a halfform bundle.

Remark 4.5. Assume that $A$ is a line bundle. Then there is a one to one correspondence between the space of Hermitian connections of $C=A^{2} \otimes K^{-1}$ and $\mathcal{D}$ given as follows: for any Hermitian connection $\nabla^{C}$ of $C$, we define first

$$
D_{X}^{A^{2}}=\nabla_{X}^{C} \otimes \mathrm{id}+\mathrm{id} \otimes D_{X}^{K}
$$

and then $D_{X}$ is the unique derivation of $\mathcal{C}^{\infty}(M, A)$ with respect to $X$ satisfying

$$
D_{X}^{A^{2}}\left(s^{2}\right)=2 s \otimes D_{X} s, \quad \forall s \in \mathcal{C}^{\infty}(M, A) .
$$

In the following proposition, we compute some kind of curvature for $D \in \mathcal{D}$.

Proposition 4.6. For any $D \in \mathcal{D}$, there exists $R \in \Omega^{2}(M, \operatorname{Herm} A)$ and a covariant derivation $\nabla: \mathcal{C}^{\infty}(M, \operatorname{Herm} A) \rightarrow \Omega^{1}(M, \operatorname{Herm} A)$ such that for any vector fields $X, Y$ of $M$

$$
\left[D_{X}, D_{Y}\right]=D_{[X, Y]}+i R(X, Y)+\frac{1}{2} B_{j}(X, Y)
$$

with $B_{j}(X, Y)$ the function defined in Lemma 4.9 and for any $f \in \mathcal{C}^{\infty}(M, \operatorname{Herm} A)$ and $s \in \mathcal{C}^{\infty}(M, A)$,

$$
D_{X}(f . s)=\left(\nabla_{X} f\right) . s+f . D_{X} .
$$

Furthermore, the curvature of $\nabla$ is $i \operatorname{ad}_{R}, \nabla R=0, \nabla \mathrm{id}=0$ and for any $f, g \in \mathcal{C}^{\infty}(M$, End $A), \nabla(f . g)=(\nabla f) . g+f .(\nabla g)$.

Proof. Assume first as in Remark 4.4 that $A=B \otimes \delta$ and $D_{Y}=\nabla_{Y}^{B} \otimes$ $\mathrm{id}+\mathrm{id} \otimes D_{Y}^{\delta}$. Then we have a natural identification $\operatorname{Herm} A \simeq \operatorname{Herm} B$. We set $R=\frac{1}{i} \operatorname{courb} \nabla^{B}$. Equation (25) follows from Lemma 4.2 We define $\nabla$ as the connection of Herm $B$ induced by $\nabla^{B}$, so that Equation (26) is satisfied and the properties of $\nabla$ given in the proposition are standard properties.

We can now extend to the result to the case where there is no half-form bundle. Observe first that $R$ and $\nabla$ are uniquely determined by Equations (25) and (26). Since this unicity is also local, the local existence of $R$ and $\nabla$ implies their global existence. But each point of $M$ has a neighborhood admitting a half-form bundle, where we can apply the first part of the proof. 


\section{The quantization map}

Consider $D \in \mathcal{D}$. For any $f \in \mathcal{C}^{\infty}(M)$, define the derivative $P_{f, k}$ in the direction of the Hamiltonian vector field $X$ of $f$

$$
P_{f, k}=\left(\nabla_{X}^{L^{k}} \otimes \mathrm{id}+\mathrm{id} \otimes D_{X}\right): \mathcal{C}^{\infty}\left(M, L^{k} \otimes A\right) \rightarrow \mathcal{C}^{\infty}\left(M, L^{k} \otimes A\right) .
$$

Then we set

$$
Q_{k}^{D}(f)=\Pi_{k}\left(f+\frac{i}{k} P_{f, k}\right): \mathcal{H}_{k} \rightarrow \mathcal{H}_{k}
$$

Theorem 5.1. Let $D \in \mathcal{D}$.

1. For any $f \in \mathcal{C}^{\infty}(M),\left(Q_{k}^{D}(f)\right)$ is a Toeplitz operator with principal symbol $f$.

2. For any $f$ and $g \in \mathcal{C}^{\infty}(M)$, we have

$$
Q_{k}^{D}(f) Q_{k}^{D}(g)=Q_{k}^{D}(f g)+\frac{1}{2 i k} \Pi_{k}\{f, g\}+\mathcal{O}\left(k^{-2}\right)
$$

and

$$
i k\left[Q_{k}^{D}(f), Q_{k}^{D}(g)\right]=Q_{k}^{D}(\{f, g\})+\frac{1}{k} \Pi_{k} R(X, Y)+\mathcal{O}\left(k^{-2}\right),
$$

where $X$ and $Y$ are the Hamiltonian vector field of $f$ and $g$ respectively and $R \in \Omega^{2}(M, \operatorname{Herm} A)$ is defined as in Proposition 4.6 .

3. For any $f \in \mathcal{C}^{\infty}(M)$ and Toeplitz operator $S,\left(k i\left[Q_{k}^{D}(f), S\right]\right)$ is a Toeplitz operator with principal symbol

$$
\sigma_{\mathrm{p}}\left(k i\left[Q_{k}^{D}(f), S\right]\right)=-\nabla_{X}\left(\sigma_{\mathrm{p}}(S)\right) .
$$

where $\nabla$ is the connection of Herm $A$ defined in Proposition 4.6 and $X$ is the Hamiltonian vector field of $f$.

The theorem will be proved in Section 7 . We can now defined a subprincipal symbol map $\sigma_{\mathrm{s}}^{D}: \mathcal{T}_{\mathrm{sc}} \rightarrow \mathcal{C}^{\infty}(M$, End $A)$ by

$$
\sigma_{\mathrm{s}}^{D}(T):=\sigma_{\mathrm{p}}\left(k\left(T-Q^{D}\left(\sigma_{\mathrm{p}}(T)\right)\right)\right), \quad \forall T \in \mathcal{T}_{\mathrm{sc}}
$$

so that we have

$$
T=Q^{D}(f)+k^{-1} \Pi g \text { modulo } k^{-2} \mathcal{T} \quad \text { with } f=\sigma_{\mathrm{p}}(T) \text { and } g=\sigma_{\mathrm{s}}^{D}(T) .
$$

Corollary 5.2. For any $D \in \mathcal{D}, \sigma_{\mathrm{s}}^{D}$ belongs to $\mathcal{E}$. The two form $R$ and the connection $\nabla$ corresponding to $\sigma_{\mathrm{s}}^{D}$, cf. Proposition 3.3, are the ones corresponding to $D$, cf. Proposition 4.6. Furthermore the map sending $D$ into $\sigma_{\mathrm{s}}^{D}$ is an affine space isomorphism from $\mathcal{D}$ to $\mathcal{E}$.

Proof. $\sigma_{\mathrm{s}}^{D}$ clearly satisfies Conditions i) and ii) Condition iii) follows from the first equation of the second assertion of Theorem 5.1. The fact that the two form $R$ and the covariant derivative $\nabla$ corresponding to $D$ and $\sigma_{\mathrm{s}}^{D}$ are the same follows from the second and third assertion of Theorem 5.1 Finally, recall that both $\mathcal{D}$ and $\mathcal{E}$ are affine spaces directed respectively by $\Omega^{1}(M, \operatorname{Herm} A)$ and $\mathcal{C}^{\infty}(M, T M \otimes \operatorname{Herm} A)$, cf. Propositions 3.2, 4.3. These vector spaces are isomorphic through $\omega$. The map sending $D$ to $\sigma_{\mathrm{s}}^{D}$ is a morphism of affine spaces. 


\section{Schwartz kernel of Toeplitz operators}

\section{The algebra $\mathcal{A}_{0}$}

We briefly recall the definition and properties of the algebra $\mathcal{A}_{0}$. The reader is referred to Cha15 for a more detailed exposition. $\mathcal{A}_{0}$ depends on the data $M, L, A, j$. By definition, $\mathcal{A}_{0}$ consists of families $\left(P_{k}: \mathcal{C}^{\infty}\left(M, L^{k} \otimes A\right) \rightarrow\right.$ $\left.\mathcal{C}^{\infty}\left(M, L^{k} \otimes A\right), k \in \mathbb{N}^{*}\right)$ of operators whose Schwartz kernels are smooth and of the following form: for any $N$, we have uniformly on $M^{2}$

$$
P_{k}(x, y)=\left(\frac{k}{2 \pi}\right)^{n} E^{k}(x, y) \sum_{\ell \in \mathbb{Z} \cap[-N, N / 2]} k^{-\ell} f_{\ell}(x, y)+\mathcal{O}\left(k^{n-(N+1) / 2}\right) .
$$

where $E$ and the $f_{\ell}$ 's are sections of $E \otimes \bar{E}$ and $A \otimes \bar{A}$ respectively which satisfy the following conditions. For any $x, y \in M, E(x, x)=1,|E(x, y)|<1$ if $x \neq y$ and $\bar{E}(x, y)=E(y, x)$. For any $Z \in \mathcal{C}^{\infty}\left(M, T^{1,0} M\right), \nabla_{(\bar{Z}, 0)} E$ vanishes to second order along the diagonal of $M^{2}$. For any negative $\ell, f_{\ell}$ vanishes to order $-3 \ell$ along the diagonal. It is also required that the successive derivatives of $P_{k}(x, y)$ are uniformly slowly increasing as $k$ tends to $\infty$, cf. Section 2.2 of [Cha15] for a more precise formulation.

For any $m \in \mathbb{N}$, define $\mathcal{A}_{m}$ as the subspace of $\mathcal{A}_{0}$ consisting of families with a Schwartz kernel in $\mathcal{O}\left(k^{n-m / 2}\right)$. By Theorem 3.3 of Cha15, $\mathcal{A}_{0}$ is an algebra and $\mathcal{A}_{m} \cdot \mathcal{A}_{p} \subset \mathcal{A}_{m+p}$ for any $m$ and $p$. Furthermore, we can describe the quotients $\mathcal{A}_{m} / \mathcal{A}_{m+1}$ by a convenient symbol and compute the corresponding products as follows. First $\left(P_{k}\right) \in \mathcal{A}_{m}$ if and only if in the asymptotic expansion (29), for any $\ell$ such that $-m \leqslant \ell \leqslant m / 2$, the coefficient $f_{\ell}$ vanishes to order $m-2 \ell$ along the diagonal. If it is the case, the symbol of $\left(P_{k}\right)$ is defined by

$$
\sigma_{m}\left(P_{k}\right)=\sum_{\ell \in \mathbb{Z} \cap[-m, m / 2]} \hbar^{\ell}\left[f_{\ell}\right]
$$

where $\left[f_{\ell}\right] \in \mathcal{C}^{\infty}\left(M, S^{m-2 \ell}\left(T^{*} M\right) \otimes\right.$ End $\left.A\right)$ is the linearization of $f_{\ell}$ along the diagonal at order $m-2 \ell$. More explicitly, if $\partial_{1}, \ldots, \partial_{n}$ is a local frame of $T^{1,0} M$ and $\left(z_{i}\right)$ is the dual frame of $\left(T^{1,0} M\right)^{*}$, we set

$$
\left[f_{\ell}\right](z, \bar{z})=\left.\sum_{|\alpha|+|\beta|=m-2 \ell} \frac{1}{\alpha ! \beta !}\left(\left(\bar{\partial}^{\beta} \otimes \partial^{\alpha}\right) f_{\ell}\right)\right|_{\operatorname{diag} M} z^{\alpha} \bar{z}^{\beta} .
$$

Then clearly $\sigma_{m}\left(P_{k}\right)=0$ if and only if $\left(P_{k}\right) \in \mathcal{A}_{m+1}$. Furthermore if $\left(P_{k}\right) \in \mathcal{A}_{p}$ and $\left(Q_{k}\right) \in \mathcal{A}_{q}$ then the symbol of $\left(P_{k} Q_{k}\right) \in \mathcal{A}_{p+q}$ is equal to $\sigma_{p}(P) \star \sigma_{q}(Q)$ where $\star$ is given by

$$
(e \star g)(\hbar, z, \bar{z})=[\exp (\hbar \Delta)(e(\hbar,-u, \bar{z}+\bar{u}) g(\hbar, z+u,-\bar{u}))]_{u=\bar{u}=0}
$$

In this formula, $\Delta=\sum \partial_{i} \bar{\partial}_{i}$ acts on the variables $u, \bar{u}$. 


\section{Toeplitz operators}

Denote by $\Pi_{k}$ the orthogonal projector of $\mathcal{C}^{\infty}\left(M, L^{k} \otimes A\right)$ onto $\mathcal{H}_{k}$. Recall that the family $\left(\mathcal{H}_{k}\right)$ is chosen in such a way that $\left(\Pi_{k}\right)$ belongs to $\mathcal{A}_{0}$ and has symbol $\sigma_{\mathrm{p}}(\Pi)=1_{A}$. We have the following characterization of Toeplitz operators:

$$
T \in \mathcal{T} \quad \Leftrightarrow \quad T \in \mathcal{A}_{0} \text { and } \Pi T \Pi=T .
$$

This in particular gives a description of the Schwartz kernel of a Toeplitz operator. Furthermore, for any $T \in \mathcal{T}$ and $m \in \mathbb{N}, \sigma_{\text {cont }}(T)=\mathcal{O}\left(\hbar^{m}\right)$ if and only if $T \in \mathcal{A}_{2 m}$. If it is the case, we have $\sigma_{2 m}(T)=\hbar^{m} f$ and $\sigma_{\text {cont }}(T)=$ $\hbar^{m} f+\mathcal{O}\left(\hbar^{m+1}\right)$ for the same section $f \in \mathcal{C}^{\infty}(M$, End $A)$. Another useful property is that for any odd $p, \mathcal{A}_{p} \cap \mathcal{T}=\mathcal{A}_{p+1} \cap \mathcal{T}$.

Introduce a vector field $X$ of $M$ and a derivative $D_{X}: \mathcal{C}^{\infty}(M, A) \rightarrow \mathcal{C}^{\infty}(M, A)$ in the direction of $X$ preserving the metric of $A$. Denote by $P_{X, k}$ the derivative

$$
P_{X, k}=\left(\nabla_{X}^{L^{k}} \otimes \mathrm{id}+\mathrm{id} \otimes D_{X}\right): \mathcal{C}^{\infty}\left(M, L^{k} \otimes A\right) \rightarrow \mathcal{C}^{\infty}\left(M, L^{k} \otimes A\right) .
$$

Lemma 6.1. The operator family $\left(\frac{i}{k} P_{X, k} \Pi_{k}\right)$ belongs to $\mathcal{A}_{1}$. Its symbol is the section $\tau_{Y} 1_{A}$ where $\tau_{Y} \in \mathcal{C}^{\infty}\left(M, T^{*} M\right)$ is given by $\tau_{Y}=\omega\left(\cdot, Y^{1,0}\right)$.

Proof. The Schwartz kernel of $\frac{i}{k} P_{X, k} \Pi_{k}$ being $\left(\frac{i}{k} P_{X, k} \otimes \mathrm{id}\right) \Pi_{k}$, the result is a particular case of Lemma 2.19 in Cha15.

Lemma 6.2. Assume that $X$ is the Hamiltonian vector field of $f \in \mathcal{C}^{\infty}(M)$. Then the family $\left(\left[f+\frac{i}{k} P_{X, k}, \Pi_{k}\right]\right)$ belongs to $\mathcal{A}_{2}$. Using the same notations as in Equation (30), its symbol is

$$
\left(\sum_{i, j=1}^{n} \omega\left(\bar{\partial}_{i},\left[\bar{\partial}_{j}, X\right]\right) \bar{z}_{i} \bar{z}_{j}-\omega\left(\partial_{i},\left[\partial_{j}, X\right]\right) z_{i} z_{j}\right) 1_{A} .
$$

This is a particular case of Lemma 3.5 in [Cha15. Let us deduce a first consequence of Lemma 6.1 Consider a second vector field $Y$ of $M$ and a derivation $D_{Y}$ of $\mathcal{C}^{\infty}(M, A)$. Let $P_{Y, k}$ be the corresponding operator.

Lemma 6.3. The operator family $\left(\frac{1}{k^{2}} \Pi_{k} P_{X, k} P_{Y, k} \Pi_{k}\right)$ belongs to $\mathcal{A}_{2}$. Its symbol is $-i \hbar \omega\left(X^{0,1}, Y^{1,0}\right)$.

Proof. By Lemma 6.1, $\left(\frac{i}{k} P_{X, k} \Pi_{k}\right)$ belong to $\mathcal{A}_{1}$ and its symbol is $\tau_{X} 1_{A}$. Taking adjoint and using that $\left(\frac{i}{k} \Pi_{k} \operatorname{div} X\right) \in \mathcal{A}_{2}$, we get that $\left(\Pi_{k} \frac{i}{k} P_{X, k}\right)$ belongs to $\mathcal{A}_{1}$. Furthermore its symbol is $\bar{\tau}_{X} 1_{A}$. Consequently, $\left(-\frac{1}{k^{2}} \Pi_{k} P_{X, k} P_{Y, k} \Pi_{k}\right)$ belongs to $\mathcal{A}_{2}$. By Equation (31), its symbol is

$$
\bar{\tau}_{X} 1_{A} \star \tau_{Y} 1_{A}=\hbar \sum \omega\left(\partial_{i}, X^{0,1}\right) \omega\left(\bar{\partial}_{i}, Y^{1,0}\right)
$$

where $\left(\partial_{i}\right)$ is an orthonormal frame of $T^{1,0} M$. Since $i X^{0,1}=\sum \omega\left(\partial_{i}, X^{0,1}\right) \bar{\partial}_{i}$, we obtain that $\bar{\tau}_{X} 1_{A} \star \tau_{Y} 1_{A}=i \hbar \omega\left(X^{0,1}, Y^{1,0}\right)$. 


\section{Proof of Theorem 5.1}

Consider $D \in \mathcal{D}$ as in section 4.2. Recall that for any function $f \in \mathcal{C}^{\infty}(M)$, $Q_{k}^{D}(f)=\Pi_{k}\left(f+\frac{i}{k} P_{f, k}\right) \Pi_{k}$ where $P_{f, k}=\nabla_{X_{f}}^{L^{k}} \otimes \mathrm{id}+\mathrm{id} \otimes D_{X_{f}}$.

The fact that $Q_{k}^{D}(f)$ is a Toeplitz operator with principal symbol $f$ was already observed in Cha15. Let us recall the proof since it is an easy consequence of Lemma 6.1. Since $\left(\frac{i}{k} P_{X, k} \Pi_{k}\right)$ belongs to $\mathcal{A}_{1}$, the same holds for $\left(\Pi_{k} \frac{i}{k} P_{X, k} \Pi_{k}\right)$. So $\left(\Pi_{k} \frac{i}{k} P_{X, k} \Pi_{k}\right)$ is a Toeplitz operator in $\mathcal{T} \cap \mathcal{A}_{1}=\mathcal{T} \cap \mathcal{A}_{2}$. Consequently

$$
Q_{k}^{D}(f)=\Pi_{k} f \Pi_{k} \quad \bmod \mathcal{T} \cap \mathcal{A}_{2},
$$

so that $Q^{D}(f)$ is a Toeplitz operator with principal symbol $f$. This shows the first assertion of Theorem [5.1. The third assertion of Theorem 5.1 has already been proved in Theorem 5.8 of Cha15. It remains to prove the second assertion.

Lemma 7.1. For any $f, g \in \mathcal{C}^{\infty}(M)$, we have

$$
\Pi_{k}\left(f+\frac{i}{k} P_{f, k}\right)\left(g+\frac{i}{k} P_{g, k}\right) \Pi_{k}=\Pi_{k}\left(f g+\frac{i}{k} P_{f g, k}+\frac{i}{2 k} X . g\right) \Pi_{k} \quad \bmod \mathcal{A}_{4} \cap \mathcal{T} .
$$

with $X$ the Hamiltonian vector field of $f$.

Proof. Let $X$ and $Y$ be the Hamiltonian vector fields of $f$ and $g$ respectively. Then the Hamiltonian vector field of $f g$ is $f Y+g X$. Using Condition (23), we obtain that

$$
\begin{aligned}
P_{f g, k} & =f P_{g, k}+g P_{f, k}+\frac{1}{2}\left(d f\left(Y^{1,0}\right)+d g\left(X^{1,0}\right)\right) \\
& =f P_{g, k}+g P_{f, k}+\frac{1}{2}\left(d f\left(Y^{1,0}\right)+X . g-d g\left(X^{0,1}\right)\right) \\
& =f P_{g, k}+g P_{f, k}+d f\left(Y^{1,0}\right)+\frac{1}{2} X . g
\end{aligned}
$$

where we have used that

$$
-d g\left(X^{0,1}\right)=\omega\left(X^{0,1}, Y\right)=\omega\left(X^{0,1}, Y^{1,0}\right)=\omega\left(X, Y^{1,0}\right)=d f\left(Y^{1,0}\right)
$$

because $T^{1,0} M$ and $T^{0,1} M$ are Lagrangian. So we have on the one hand that

$$
f g+\frac{i}{k} P_{f g, k}+\frac{i}{2 k} X . g=f g+\frac{i}{k}\left(f P_{g, k}+g P_{f, k}+X . g\right)+\frac{i}{k} d f\left(Y^{1,0}\right) .
$$

On the other hand, we have

$$
\left(f+\frac{i}{k} P_{f, k}\right)\left(g+\frac{i}{k} P_{g, k}\right)=f g+\frac{i}{k}\left(g P_{f, k}+f P_{g, k}+X . g\right)-\frac{1}{k^{2}} P_{f, k} P_{g, k}
$$

Clearly $\Pi \frac{i}{k} d f\left(Y^{1,0}\right) \Pi$ belongs to $\mathcal{A}_{2}$. Its symbol is $i \hbar d f\left(Y^{1,0}\right)$. By Lemma 6.3. $\left(-\Pi_{k} \frac{1}{k^{2}} P_{f, k} P_{g, k} \Pi_{k}\right) \in \mathcal{A}_{2}$ and has the same symbol. So

$$
\Pi_{k} \frac{i}{k} d f\left(Y^{1,0}\right) \Pi_{k}=-\Pi_{k} \frac{1}{k^{2}} P_{f, k} P_{g, k} \Pi_{k} \quad \bmod \mathcal{A}_{3} .
$$

Recall that $\mathcal{A}_{3} \cap \mathcal{T}=\mathcal{A}_{4} \cap \mathcal{T}$. So Equations (32) and (33) imply the result. 
Theorem 7.2. For any $f, g \in \mathcal{C}^{\infty}(M)$, we have

$$
Q_{k}^{D}(f) Q_{k}^{D}(g) \equiv Q_{k}^{D}(f g)+\frac{1}{2 k i} Q_{k}^{D}(\{f, g\}) \quad \bmod \mathcal{A}_{4} \cap \mathcal{T}
$$

where $\{f, g\}$ denotes the Poisson bracket of $f$ and $g$.

Proof. Introduce the operators

$$
S(f, g, k)=\Pi_{k}\left[f+\frac{i}{k} P_{f, k}, \Pi_{k}\right]\left[g+\frac{i}{k} P_{g, k}, \Pi_{k}\right] \Pi_{k}
$$

By Lemma 6.2 the family $(S(f, g, k))$ belongs to $\mathcal{A}_{4} \cap \mathcal{T}$. A straightforward computations shows that

$$
S(f, g, k)=Q_{k}^{D}(f) Q_{k}^{D}(g)-\Pi_{k}\left(f+\frac{i}{k} P_{f, k}\right)\left(g+\frac{i}{k} P_{g, k}\right) \Pi_{k}
$$

So we have

$$
\Pi_{k}\left(f+\frac{i}{k} P_{f, k}\right)\left(g+\frac{i}{k} P_{g, k}\right) \Pi_{k}=Q_{k}^{D}(f) Q_{k}^{D}(g) \quad \bmod \mathcal{A}_{4} \cap \mathcal{T} .
$$

We conclude with Lemma 6.3 .

Lemma 7.3. For any $f, g \in \mathcal{C}^{\infty}(M)$, we have

$$
\begin{aligned}
{\left[f+\frac{i}{k} P_{f, k}, g+\frac{i}{k} P_{g, k}\right]=} & \frac{1}{i k}\left(\{f, g\}+\frac{i}{k} P_{\{f, g\}, k}\right) \\
& -\frac{1}{k^{2}} \operatorname{id} \otimes\left(i R(X, Y)+\frac{1}{2} B_{j}(X, Y)\right)
\end{aligned}
$$

where $R$ is the 2-form defined in Proposition 4.6, $X$ and $Y$ are the Hamiltonian vector fields of $f$ and $g$, and $B_{j}(X, Y)$ is the function defined in Lemma 4.2.

Proof. A famous computation shows that

$$
\left[f+\frac{i}{k} \nabla_{X}^{L^{k}}, g+\frac{i}{k} \nabla_{Y}^{L^{k}}\right]=\frac{i}{k}\left(-\{f, g\}+\frac{i}{k} \nabla_{[X, Y]}^{L^{k}}\right)
$$

where $X$ and $Y$ the Hamiltonian vector fields of $f$ and $g$. The result is now a consequence of Proposition 4.6.

Lemma 7.4. For any $f, g \in \mathcal{C}^{\infty}(M), S(f, g, k)-S(g, f, k)$ belongs to $\mathcal{A}_{4}$ and its symbol is $\frac{\hbar^{2}}{2} B_{j}(X, Y)$.

Proof. By Lemma 6.2, the symbol $\sigma_{X}$ of $\left(\left[f+\frac{i}{k} P_{f, k}, \Pi_{k}\right]\right)$ has the form $f_{X}(z)-$ $\overline{f_{X}(z)}$ where $f_{X}$ is quadratic. Using this fact, a direct computation shows that

$$
1_{A} \star \sigma_{X} \star \sigma_{Y} \star 1_{A}=\frac{\hbar^{2}}{2} \sum_{i, j}\left(\partial_{i} \partial_{j} \sigma_{X}\right)\left(\bar{\partial}_{i} \bar{\partial}_{j} \sigma_{Y}\right) .
$$

where we denote by $\star$ the product given in Equation (31). By Lemma 6.2

$$
\left(\partial_{i} \partial_{j} \sigma_{X}\right)=-\omega\left(\partial_{i},\left[\partial_{j}, X\right]\right), \quad\left(\bar{\partial}_{i} \bar{\partial}_{j} \sigma_{Y}\right)=\omega\left(\bar{\partial}_{i},\left[\bar{\partial}_{j}, Y\right]\right) .
$$


Since $\left(\partial_{i}\right)$ is an orthonormal frame, the dual frame is given by $\theta_{i}=\frac{1}{i} \omega\left(\cdot, \bar{\partial}_{i}\right)$. Since $Y$ preserves $\omega$, we get $\mathcal{L}_{Y} \theta_{i}=\frac{1}{i} \omega\left(\cdot,\left[Y, \bar{\partial}_{i}\right]\right)$. Replacing $Y$ by $X$ and taking the conjugate, $\mathcal{L}_{X} \bar{\theta}_{i}=-\frac{1}{i} \omega\left(\cdot,\left[X, \partial_{i}\right]\right)$. Consequently

$$
1_{A} \star \sigma_{X} \star \sigma_{Y} \star 1_{A}=-\frac{\hbar^{2}}{2} \sum_{i, j}\left(\mathcal{L}_{X} \bar{\theta}_{i}\right)\left(\partial_{j}\right)\left(\mathcal{L}_{Y} \theta_{i}\right)\left(\bar{\partial}_{j}\right)
$$

Antisymmetrising, we get the final result.

Theorem 7.5. For $f, g \in \mathcal{C}^{\infty}(M)$, we have that

$$
\left[Q_{k}^{D}(f), Q_{k}^{D}(g)\right]=\frac{1}{i k}\left(Q_{k}^{D}(\{f, g\})+\frac{1}{k} \Pi_{k} R(X, Y) \Pi_{k}\right) \quad \bmod \mathcal{A}_{6} \cap \mathcal{T}
$$

where $X$ and $Y$ are the Hamiltonian vector fields of $f$ and $g$.

Proof. By Equation (34),

$$
S(f, g, k)-S(g, f, k)=\left[Q_{k}^{D}(f), Q_{k}^{D}(g)\right]-\Pi_{k}\left[f+\frac{i}{k} P_{f, k}, g+\frac{i}{k} P_{g, k}\right] \Pi_{k}
$$

By Lemma 7.4, the left hand side is equal to $\frac{1}{k^{2}} \Pi_{k} \frac{1}{2} B_{j}(X, Y) \Pi_{k}$ modulo $\mathcal{A}_{6} \cap \mathcal{T}$. By Lemma 7.3, the right hand side is equal to

$$
\left[Q_{k}^{D}(f), Q_{k}^{D}(g)\right]-\frac{1}{i k} Q_{k}^{D}\left(\{f, g\}+\frac{i}{k^{2}} \Pi_{k} R(X, Y) \Pi_{k}+\frac{1}{k^{2}} \Pi_{k} \frac{1}{2} B_{j}(X, Y) \Pi_{k}\right.
$$

modulo $\mathcal{A}_{6} \cap \mathcal{T}$. The result follows.

\section{References}

[BCG97] Mélanie Bertelson, Michel Cahen, and Simone Gutt. Equivalence of star products. Classical Quantum Gravity, 14(1A):A93-A107, 1997. Geometry and physics.

[BdM02] Louis Boutet de Monvel. Related semi-classical and Toeplitz algebras. In Deformation quantization (Strasbourg, 2001), volume 1 of IRMA Lect. Math. Theor. Phys., pages 163-190. de Gruyter, Berlin, 2002.

[BdMG81] L. Boutet de Monvel and V. Guillemin. The spectral theory of Toeplitz operators, volume 99 of Annals of Mathematics Studies. Princeton University Press, Princeton, NJ, 1981.

[BMS94] Martin Bordemann, Eckhard Meinrenken, and Martin Schlichenmaier. Toeplitz quantization of Kähler manifolds and $\operatorname{gl}(N), N \rightarrow \infty$ limits. Comm. Math. Phys., 165(2):281-296, 1994.

[BPU98] D. Borthwick, T. Paul, and A. Uribe. Semiclassical spectral estimates for Toeplitz operators. Ann. Inst. Fourier (Grenoble), 48(4):1189-1229, 1998. 
[Cha03a] L. Charles. Berezin-Toeplitz operators, a semi-classical approach. Comm. Math. Phys., 239(1-2):1-28, 2003.

[Cha03b] L. Charles. Quasimodes and Bohr-Sommerfeld conditions for the Toeplitz operators. Comm. Partial Differential Equations, 28(910):1527-1566, 2003.

[Cha06] L. Charles. Symbolic calculus for Toeplitz operators with half-form. J. Symplectic Geom., 4(2):171-198, 2006.

[Cha07] L. Charles. Semi-classical properties of geometric quantization with metaplectic correction. Comm. Math. Phys., 270(2):445-480, 2007.

[Cha15] L. Charles. Quantization of compact symplectic manifolds. Journal of Geometric Analysis, 2015.

[Fed96] Boris Fedosov. Deformation quantization and index theory, volume 9 of Mathematical Topics. Akademie Verlag, Berlin, 1996.

[Gui95] Victor Guillemin. Star products on compact pre-quantizable symplectic manifolds. Lett. Math. Phys., 35(1):85-89, 1995.

[Le 13] Y. Le Floch. Singular Bohr-Sommerfeld conditions for 1D Toeplitz operators: hyperbolic case. ArXiv e-prints, September 2013.

[MM07] Xiaonan Ma and George Marinescu. Holomorphic Morse inequalities and Bergman kernels, volume 254 of Progress in Mathematics. Birkhäuser Verlag, Basel, 2007.

[MM12] Xiaonan Ma and George Marinescu. Berezin-Toeplitz quantization on Kähler manifolds. J. Reine Angew. Math., 662:1-56, 2012. 